University of South Carolina

Scholar Commons

$1-7-2010$

\title{
Quantum Trajectory Dynamics in Imaginary Time with the Momentum-Dependent Quantum Potential
}

Sophya Garashchuk

University of South Carolina--Columbia, sgarashc@chem.sc.edu

Follow this and additional works at: https://scholarcommons.sc.edu/chem_facpub

Part of the Chemistry Commons

Publication Info

Published in Journal of Chemical Physics, Volume 132, Issue 1, 2010, pages 014112-.

(c) Journal of Chemical Physics 2010, American Institute of Physics.

This Article is brought to you by the Chemistry and Biochemistry, Department of at Scholar Commons. It has been accepted for inclusion in Faculty Publications by an authorized administrator of Scholar Commons. For more information, please contact digres@mailbox.sc.edu. 


\title{
Quantum trajectory dynamics in imaginary time with the momentum-dependent quantum potential
}

\author{
Sophya Garashchuk ${ }^{\mathrm{a})}$ \\ Department of Chemistry and Biochemistry, University of South Carolina, \\ Columbia, South Carolina 29208, USA
}

(Received 27 October 2009; accepted 16 December 2009; published online 7 January 2010)

\begin{abstract}
The quantum trajectory dynamics is extended to the wave function evolution in imaginary time. For a nodeless wave function a simple exponential form leads to the classical-like equations of motion of trajectories, representing the wave function, in the presence of the momentum-dependent quantum potential in addition to the external potential. For a Gaussian wave function this quantum potential is a time-dependent constant, generating zero quantum force yet contributing to the total energy. For anharmonic potentials the momentum-dependent quantum potential is cheaply estimated from the global Least-squares Fit to the trajectory momenta in the Taylor basis. Wave functions with nodes are described in the mixed coordinate space/trajectory representation at little additional computational cost. The nodeless wave function, represented by the trajectory ensemble, decays to the ground state. The mixed representation wave functions, with lower energy contributions projected out at each time step, decay to the excited energy states. The approach, illustrated by computing energy levels for anharmonic oscillators and energy level splitting for the double-well potential, can be used for the Boltzmann operator evolution. (c) 2010 American Institute of Physics. [doi:10.1063/1.3289728]
\end{abstract}

\section{INTRODUCTION}

Quantum-mechanical (QM) evolution of wave functions in imaginary time arises primarily in two types of problems. It can be used to determine low-lying energy levels and eigenfunctions, ${ }^{1}$ in particular the energy levels of highdimensional systems using Monte Carlo methods..$^{2-4}$ The approach is based on a simple fact that in the course of imaginary-time evolution

$$
\hat{H} \psi(x, \tau)=-\hbar \frac{\partial \psi(x, \tau)}{\partial \tau}, \quad \tau>0,
$$

the higher energy components of a wave function $\psi(x, \tau)$ decay faster than the lower energy components: a wave function expressed in terms of the eigenstates $\left\{\psi_{k}\right\}$ of $\hat{H}\left(E_{k}\right.$ $>0)$

$$
\hat{H} \psi_{k}(x)=E_{k} \psi_{k}(x), \quad \psi(x, \tau)=\sum_{k} c_{k} e^{-E_{k} \tau} \psi_{k}(x),
$$

decays to the lowest energy state of the same symmetry. Some recent examples are computation of the zero-point energy and tunneling splitting in malonaldehyde ${ }^{5}$ and diffusion Monte Carlo study of $\mathrm{CH}_{5}^{+}$. ${ }^{6}$ Imaginary-time evolution also appears in computation of correlation functions for complex systems in a Boltzmann (thermal) distribution, ${ }^{7}$ such as the reaction rate constants within the thermal flux operator approach. ${ }^{8}$

In high-dimensional systems the approximate methods are generally needed to propagate a wave function. The trajectory-based propagation techniques are attractive in this

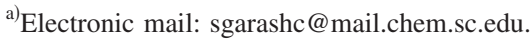

context, because they combine intuitiveness and low cost of classical mechanics with description of the leading quantum effects on dynamics. Several such approaches are based on the de Broglie-Bohm formulation of the Schrödinger equation $(\mathbf{S E})$ in terms of the quantum trajectories evolving under combined influence of the external or classical potential $V$ and the quantum potential determined approximately. ${ }^{9-14}$ Here we apply ideas of the quantum trajectory (QT) dynamics to the QM evolution in imaginary time by representing a real positive wave function, which decays to the ground state, in the exponential form. For approximate, thus scalable in principle to many dimensions, implementation we determine the imaginary-time analog of the quantum potential by fitting the trajectory momenta in a small basis. Evolution of the excited states is accomplished in the mixed wave function representation compatible with the trajectory dynamics. ${ }^{15,16}$

The de Broglie-Bohm formulation of the conventional $\mathrm{SE}$ in real time, ${ }^{17,18}$ given here in one Cartesian dimension for simplicity, is based on the polar representation of a wave function in terms of the real amplitude $A(x, t)$ and phase $S(x, t)$

$$
\psi(x, t)=A(x, t) e^{\imath S(x, t) / \hbar} .
$$

Substitution of Eq. (3) into SE

$$
\hat{H} \psi(x, t)=l \frac{\partial \psi(x, t)}{\partial t}, \quad \hat{H}=-\frac{\hbar^{2}}{2 m} \frac{\partial^{2}}{\partial x^{2}}+V,
$$

and identification of the gradient of the phase $S(x, t)$ with the momentum of a trajectory 


$$
p(x, t)=\nabla S(x, t),
$$

leads to the classical-like equations of motion of the trajectory-specific quantities

$$
\begin{aligned}
& \frac{d S_{t}}{d t}=\frac{p_{t}^{2}}{2 m}-\left.(V+U)\right|_{x=x_{t}}, \\
& \frac{d x_{t}}{d t}=\frac{p_{t}}{m}, \quad \frac{d p}{d t}=-\left.\nabla(V+U)\right|_{x=x_{t}},
\end{aligned}
$$

in the Lagrangian frame of reference

$$
\frac{d}{d t}=\frac{\partial}{\partial t}+\frac{p}{m} \frac{\partial}{\partial x} .
$$

$\nabla$ is used to denote differentiation with respect to $x$ for compactness of notations. All quantum effects come from the quantum potential $U$

$$
U=-\frac{\hbar^{2}}{2 m} \frac{\nabla^{2} A(x, t)}{A(x, t)} .
$$

Evolution of the wave function amplitude obeys the continuity equation

$$
\frac{d A^{2}\left(x_{t}\right)}{d t}=-\frac{\nabla p\left(x_{t}\right)}{m} A^{2}\left(x_{t}\right) .
$$

Equation (9) together with the evolution of the volume element associated with a trajectory located at $x_{t}$

$$
\delta x_{t}=\delta x_{0} \exp \left(\int_{0}^{t} \frac{\nabla p_{\tau}}{m} d \tau\right),
$$

gives conservation of the probability within $\delta x_{t}$ [or of the trajectory weight $\left.w\left(x_{t}\right)\right]$

$$
w\left(x_{t}\right)=A^{2}\left(x_{t}\right) \delta x_{t}, \quad \frac{d w\left(x_{t}\right)}{d t}=0 .
$$

In the numerical implementation, $\psi(x, 0)$ is represented by an ensemble of $N_{\text {traj }}$ trajectories, their positions $x_{0}^{(k)}$ $\left(k=1 \cdots N_{\text {traj }}\right)$ sampling $A(x, 0)$. Their initial momenta $p_{0}^{(k)}$ are defined from Eq. (5). For an operator $\hat{\Omega}(x)$ the average value can be computed using constant trajectory weights, $w^{(k)}$, as

$$
\langle\hat{\Omega}\rangle=\sum_{k} \hat{\Omega}\left(x_{t}^{(k)}\right) w^{(k)} .
$$

Substitution of the time variable, $t \rightarrow-l \tau(\tau>0)$, transforms the real-time SE (4) into the diffusion Eq. (1). In semiclassical treatments of Eq. (1) (Refs. 7 and 19) the trajectory equations of motion are obtained by the change $p \rightarrow-l p$ and $S \rightarrow-l S$, which yields the classical equations of motion in the "inverted" potential (the negative of the original $V$ ). For the quantum trajectories, however, the same transformation of Eq. (6) introduced by Liu and Makri, ${ }^{20}$ results in singular trajectory dynamics describing evolution of a nonsingular Gaussian wave function. Such behavior is traced to the nonunique representation of a real wave function in terms of the amplitude and "phase," as Eq. (3) becomes

$$
\psi(x, \tau)=A(x, \tau) e^{-S(x, \tau) / \hbar} .
$$

Liu and Makri ${ }^{12}$ elegantly circumvent the singularity problem by repartitioning $\psi(x, \tau)$ between the amplitude and phase factors in a way that produces smooth-essentially stationary-trajectories. The formulation implemented with independent approximate trajectories using their stability properties gives accurate ground state vibrational energies in one-three dimensions by evolving a single trajectory, its stability matrix elements and their derivatives up to sixth order. Goldfarb et $_{\mathrm{al}}{ }^{21}$ introduced the zero-velocity complex action method (ZEVCA), which, in imaginary time, is similar to the approach of Liu and Makri. ZEVCA is based on the exponential form of the wave function without the amplitude factor $A(x, \tau)$ in Eq. (13), and it postulates "zero-velocity trajectories." Then, SE is solved around a fixed $x$-value by evolving derivatives of $S(x, \tau)$ in the Eulerian frame of reference. One-dimensional examples in Ref. 21 give the energy accuracy, numerical cost, and the trajectory behavior similar to those of Liu and Makri.

We believe that while stationary or nearly stationary trajectories work well and might be advantageous for the ground vibrational energies, identification of the trajectory momentum with the action function $S(x, \tau)$ (and trajectory motion) is essential part of the definition of the quantum trajectories. Such trajectories follow changes in the wave function density in the course of evolution-a feature important for efficient description of large amplitude motion or reactive dynamics. Therefore, we develop the imaginarytime QT formulation using an unambiguous representation of a real positive wave function as a simple exponent

$$
\psi(x, \tau)=e^{-S(x, \tau) / \hbar},
$$

and use the Bohmian definition of the trajectory momentum of Eq. (5). Section II presents the new theory. Application to anharmonic potentials and discussion are given in Sec. III. Section IV concludes.

\section{IMAGINARY TIME PROPAGATION}

\section{A. The quantum trajectory dynamics in imaginary time}

Substitution of the exponential wave function (14) into Eq. (1) and division by $\psi(x, \tau)$ gives

$$
\frac{\partial S(x, \tau)}{\partial \tau}=-\frac{1}{2 m}\left(\frac{\partial S(x, \tau)}{\partial x}\right)^{2}+V+\frac{\hbar}{2 m} \nabla^{2} S(x, \tau) .
$$

Using $\tau$ as a time variable and defining the trajectory momentum by Eq. (5), Eq. (15) in the Lagrangian frame of reference given by Eq. (7) leads to the following equations of motion:

$$
\frac{d x}{d \tau}=\frac{p}{m}, \quad \frac{d p}{d \tau}=\nabla(V+U) .
$$

Compared with the real-time Eqs. (6) and (8), in imaginary time we get the inverted potential and a different definition of the quantum potential 


$$
U(x, \tau)=\frac{\hbar}{2 m} \nabla^{2} S=\frac{\hbar \nabla p}{2 m} .
$$

Note that $U$ of Eq. (17) is proportional to $\hbar / m$ and, thus, vanishes in the classical limit. In the multidimensional case Eq. (17) generalizes to $U(\vec{x}, \tau)=\hbar \vec{\nabla} \cdot \vec{\nabla} S /(2 m)$. The "classical" evolution can be defined by setting $U=0$. Evolution of the imaginary-time action function is

$$
\frac{d S}{d \tau}=\frac{p^{2}}{2 m}+V+U
$$

and the energy of a trajectory is

$$
\varepsilon=\frac{\partial S}{\partial \tau}=-\frac{p^{2}}{2 m}+V+U
$$

Remarkably, for the wave function (14) evolution of the volume element $\delta x_{\tau}$ given by Eq. (10) cancels contribution of the quantum potential of Eq. (17) to the average quantities,

$$
\langle\Omega\rangle=\int \Omega(x) e^{-2 S(x) / \hbar} d x=\sum_{k} \Omega\left(x_{k}\right) e^{-2 \tilde{S}_{k} / \hbar} \delta x_{0}^{(k)} .
$$

$\delta x_{0}^{(k)}$ is a volume element associated with $k^{\text {th }}$ trajectory at time $\tau=0 . \tilde{S}$ indicates the classical action function computed along the quantum trajectory,

$$
\tilde{S}_{\tau}=\int_{0}^{\tau}\left(\frac{p_{t}^{2}}{2 m}+\left.V(x)\right|_{x=x_{t}}\right) d t .
$$

Integration over $x$ goes from $x=-\infty$ to $x=\infty$ in Eq. (20) and below. Planck's constant in atomic units, $\hbar=1$, is used below.

For a Gaussian wave function evolving in a quadratic potential, $S(x, \tau)$ is a quadratic function of $x, p(x, \tau)$ is linear in $x, U$ is a time-dependent constant and the quantum force, $\nabla U$, is zero. Therefore, average $x$ - or $p$-dependent quantities of classical $(U=0)$ and $\mathrm{QM}$ evolutions are the same. This quantum/classical similarity was one of the motivations behind the real-time Bohmian mechanics with complex action, ${ }^{22}$ based on the wave function form $\psi(x, t)$ $=\exp \left({ }_{i} S_{Z}(x, t)\right)$. Despite the conceptual appeal real-time complex $S_{Z}(x, t)$ is responsible for a very challenging implementation of dynamics in complex $(x, p)$ space.

The imaginary-time dynamics governed by Eqs. (16)-(18) leads to the following properties of the trajectory ensemble. (i) The average momentum remains zero

$$
\langle p\rangle=\int e^{-S}(\nabla S) e^{-S} d x=-\frac{1}{2} \int d\left(e^{-2 S}\right)=0 .
$$

(ii) The time-dependence of normalization $N$

$$
N(\tau)=\int e^{-2 S(x, \tau)} d x
$$

using Eqs. (15), (10), and (21) is proportional to the energy of trajectory ensemble

$$
\begin{aligned}
\frac{d N}{d \tau} & =-2 \int \frac{\partial S}{d \tau} e^{-2 S} d x \\
& =-2 \int\left(-\frac{p^{2}}{2 m}+V+U\right) e^{-2 S} d x=-2\langle\varepsilon\rangle
\end{aligned}
$$

(iii) The time-dependence of the total un-normalized energy E

$$
E=\langle\psi|\hat{H}| \psi\rangle=\int\left(-\frac{p^{2}}{2 m}+V+U\right) e^{-2 S} d x=\langle\varepsilon\rangle,
$$

using Eqs. (16) and (15) is

$$
\begin{aligned}
\frac{d E}{d \tau} & =\int\left(-\frac{p}{m} \frac{d p}{d \tau}+\nabla(V+U) \frac{d x}{d \tau}-2 \frac{\partial S}{\partial \tau} \varepsilon\right) e^{-2 S} d x \\
& =-2 \int \varepsilon^{2} e^{-2 S} d x=-2\left\langle\varepsilon^{2}\right\rangle .
\end{aligned}
$$

The right-hand-sides of Eqs. (24)-(26) in terms of trajectories are obtained from Eq. (20).

\section{B. Approximate implementation and excited states}

To implement Eqs. (16) and (18) one has to know the first and the second derivatives of $p$ determining $U$ and $\nabla U$. Our goal is a cheap approximate methodology. Thus, we find derivatives from the least-squares fit ${ }^{23}$ to $p(x, \tau)$ with $\psi^{2}(x, \tau)$ as a weighting function. If $p(x, \tau)$ is approximated as $p(x, \tau) \approx \vec{f}(x) \cdot \vec{b}(\tau)$ in a small basis $\vec{f}(x)$ of the size $N_{b}$, then the optimal values of the coefficients $\vec{b}$ minimizing $\left\langle(p-\vec{f} \cdot \vec{b})^{2}\right\rangle$ are

$$
\vec{b}=\mathbf{M}^{-1} \vec{P} .
$$

$\mathbf{M}$ is a symmetric matrix of the time-dependent basis function overlaps,

$$
M_{i j}=\left\langle f_{i} \mid f_{j}\right\rangle=\sum_{k} f_{i}\left(x_{\tau}^{(k)}\right) f_{j}\left(x_{\tau}^{(k)}\right) \exp \left(-2 \widetilde{S}_{\tau}^{(k)}\right) \delta x_{0}^{(k)} .
$$

The vector $\vec{P}$ includes the trajectory momenta averaged with the basis functions,

$$
P_{i}=\left\langle p \mid f_{i}\right\rangle=\sum_{k} p_{\tau}^{(k)} f_{i}\left(x_{\tau}^{(k)}\right) \exp \left(-2 \widetilde{S}_{\tau}^{(k)}\right) \delta x_{0}^{(k)} .
$$

Placing the minimum of $V$ at $x=0$ we use the Taylor basis to determine $U$ and to represent wave functions with nodes,

$$
\vec{f}(x)=\left(1, x, x^{2}, \ldots\right)^{T} .
$$

Evolution of wave functions with nodes, such as excited states functions, can be efficiently done in the mixed coordinate space/trajectory representation, ${ }^{15}$

$$
\phi(x, \tau)=\chi(x, \tau) \psi(x, \tau) .
$$

The nodeless $\psi(x, \tau)$ solves Eq. (1) and evolves in imaginary time into the ground state wave function according to Eq. (2). Substitution of Eq. (31) into Eq. (1) and division by $\psi(x, \tau)$ gives evolution of $\chi(x, \tau)(\hbar$ included $)$ as 


$$
-\frac{\partial \chi}{\partial \tau}=-\frac{\hbar^{2}}{2 m}\left(\psi \nabla^{2} \chi+2 \nabla \chi \nabla \psi\right)
$$

Representing $\chi(x, \tau)$ in the basis $\vec{f}(x)$ of size $N_{c}$,

$$
\chi(x, \tau)=\vec{f}(x) \cdot \vec{c}(\tau),
$$

evolution of the coefficients $\vec{c}$ is obtained by multiplying Eq. (32) by $\psi(x, \tau) \vec{f}(x)$ and integrating by parts. Defining the matrix $\mathbf{D}$ of the basis functions derivatives,

$$
D_{i j}=\left\langle\nabla f_{i} \mid \nabla f_{j}\right\rangle=\sum_{k} \nabla f_{i}\left(x_{\tau}^{(k)}\right) \nabla f_{j}\left(x_{\tau}^{(k)}\right) \exp \left(-2 \widetilde{S}_{\tau}^{(k)}\right) \delta x_{0}^{(k)},
$$

the evolution of $\vec{c}(\tau)$ is

$$
\frac{d \vec{c}}{d \tau}=-\frac{\hbar}{2 m} \mathbf{M}^{-1} \mathbf{D} \vec{c} .
$$

It can be shown that the approximate evolution of $\chi$ and $\psi$ leads to the correct time-dependence of the normalization for the total wave function $\phi(x, \tau)$,

$$
\frac{d\langle\phi \mid \phi\rangle}{d \tau}=-2\langle\phi|\hat{H}| \phi\rangle=-2 \int\left(\varepsilon|\chi|^{2}+\frac{|\nabla \chi|^{2}}{2 m}\right) d x=-2 E .
$$

To find the ground state of a system one can start with an arbitrary trial wave function without nodes. We choose $\psi(x, 0)$ as a Gaussian wave function, since its evolution is exact in a quadratic potential for a linear basis, $N_{b}=2$. Although linear approximation to $p(x, \tau)$ gives zero quantum force and, thus, does not affect positions of the trajectories, the quantum potential is still needed to define the total energy.

To obtain $N_{s}$ eigenstates we evolve $N_{s}$ coordinatespace functions $\chi_{n}, n=1 \ldots N_{s}$, represented in the Taylor basis (30). The coefficients can be written as a matrix $\mathbf{C}=\left(\vec{c}_{1}, \vec{c}_{2} \ldots, \vec{c}_{N_{s}}\right)$. The number of states, $N_{s}$, is no larger than the basis size, $N_{c}$. The initial functions $\chi_{j}$ will have correct number of nodes, if the initial values $C_{i j}(0)$ are chosen, for example, as the Hermite polynomial coefficients of the harmonic oscillator eigenfunction. At each time step wave functions of the lower energy are projected out

$$
\chi_{j}^{\text {new }}=\chi_{j}-\sum_{k, k<j}\left\langle\chi_{j} \mid \chi_{k}\right\rangle \chi_{k} .
$$

In terms of $C_{i j}$ and the overlap matrix (28) the projection (37) is

$$
C_{i j}^{\text {new }}=C_{i j}-\sum_{k, k<j}\left\langle\chi_{j} \mid \chi_{k}\right\rangle C_{i k}, \quad\left\langle\chi_{j} \mid \chi_{k}\right\rangle=\left(\mathbf{C}^{T} \mathbf{M C}\right)_{j k} .
$$

Both determination of $\vec{b}$ defining $U$ and evolution of $\mathbf{C}$, require inversion of the overlap matrix $\mathbf{M}$. Thus, if $N_{c}=N_{b}$, then the calculation of the excited states requires little effort in addition to the QT propagation. The outlined approach is approximate and is expected to work for a few low energy eigenstates where modest basis size is reasonable. In the

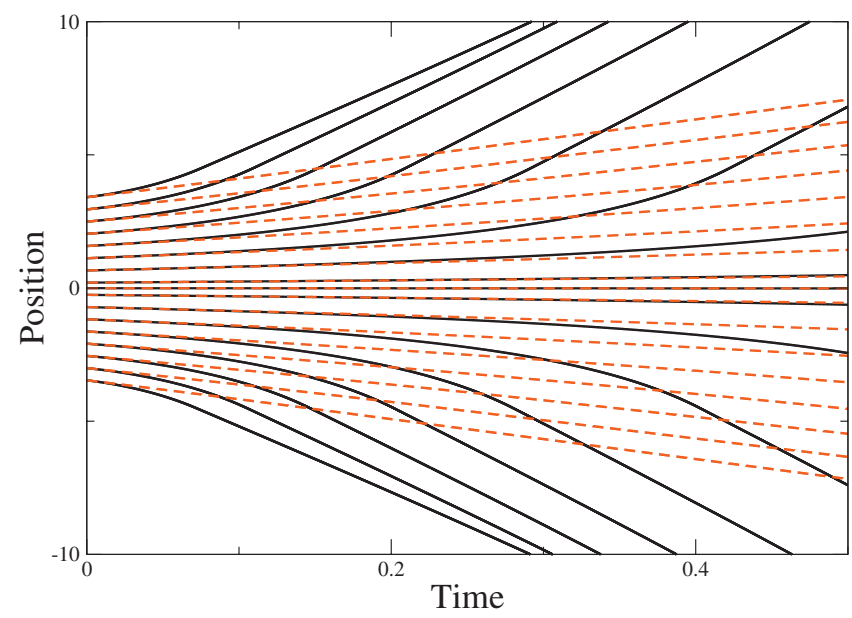

FIG. 1. Imaginary-time quantum trajectories for the quadratic (dash) and quartic (solid line) oscillators. The quantum potential is found approximately using $N_{b}=4$ for the latter potential.

examples below we compute four eigenstates of onedimensional anharmonic systems using up to six basis functions.

\section{EXAMPLES AND DISCUSSION}

To illustrate the imaginary-time QT method with approximate momentum-dependent quantum potential we consider several anharmonic systems, which proved to be very challenging for the real-time quantum trajectories in exact or approximate implementations. ${ }^{24-26}$ The difficulty is the inherent instability of the real-time Bohmian trajectories describing stationary eigenstates: such trajectories should not move, therefore classical and quantum forces have to cancel each other exactly. If this is not the case, even small displacements of the trajectory positions in the course of dynamics lead to rapid "decoherence" of the quantum trajectories and, consequently, to the loss of quantum effects. In contrast, the imaginary-time trajectories are not stationaryheir dynamics reflects decay of the wave function. With the functional form of Eq. (14) the trajectories describing imaginary-time evolution of a Gaussian are nonsingular: they simply "roll off" a parabolic barrier, i.e., off the inverted well centered at $x=0$, as shown on Fig. 1. The same is true for anharmonic single-well potentials in one dimensions.

Two single-well potentials from Ref. 20 were considered-a well with a large quartic anharmonicity and the Morse oscillator modeling the hydrogen molecule. Potential with quartic anharmonicity is

$$
V=\frac{x^{2}}{2}+x^{4}
$$

and the mass of the particle is $m=1$ a.u. The initial wave function was taken as a Gaussian

$$
\psi(x, 0)=\left(\frac{2 a}{\pi}\right)^{1 / 4} e^{-a\left(x-x_{0}\right)^{2}}
$$

with the width parameter $a=1.0 a_{0}^{-2}$ and $x_{0}=0 a_{0} .500$ trajectories were propagated up to $\tau=1.0$ a.u. The trajectories corresponding to $V$ of Eq. (39) and its quadratic approximation, 
TABLE I. The lowest energy levels $(n=0,1,2,3)$ for the quartic oscillator given by Eq. (39) obtained from the imaginary-time QT dynamics. The basis sizes in the approximations of $U$ and $\chi$ are given in the first two columns, respectively. The bottom lines contain the exact QM values from the Hamiltonian matrix diagonalization and analytical values in the quadratic approximation to the potential.

\begin{tabular}{lccccc}
\hline \hline$N_{b}$ & $N_{c}$ & \multicolumn{1}{c}{$E_{0}$} & \multicolumn{1}{c}{$E_{1}$} & \multicolumn{1}{c}{$E_{2}$} & \multicolumn{1}{c}{$E_{3}$} \\
\hline 2 & 4 & 0.76007 & 2.3139 & 4.5298 & 7.1670 \\
2 & 6 & 0.76007 & 2.3147 & 4.5718 & 7.1919 \\
4 & 4 & 0.80358 & 2.7371 & 5.2856 & 8.2111 \\
4 & 6 & 0.80358 & 2.7373 & 5.2245 & 8.0436 \\
6 & 4 & 0.80375 & 2.7381 & 5.2333 & 8.0762 \\
6 & 6 & 0.80375 & 2.7379 & 5.1829 & 7.9435 \\
$\mathrm{QM}$ & & 0.80377 & 2.7379 & 5.1719 & 7.9424 \\
$\mathrm{HO}$ & & 0.5 & 1.5 & 2.5 & 3.5 \\
\hline \hline
\end{tabular}

$V^{\text {quad }}=x^{2} / 2$, are shown on Fig. 1 . The quantum potential was computed using four basis functions in the case of full $V$. The trajectories spread out faster in the anharmonic case, but the overall behavior is similar to the dynamics in the quadratic potential.

Four lowest energy levels and wave functions were computed using bases of the size $N_{b}=\{2,4,6\}$ and using $N_{c}=4$ and $N_{c}=6$ polynomials to represent $\chi_{n}$ for $n=\{0,1,2,3\}$. We did not use the symmetry of the system in the calculation. The initial values of the polynomial coefficients forming matrix $\mathbf{C}$ were taken as for the excited states of the harmonic oscillator with the ground state given by Eq. (40). The ground state energy of the quartic oscillator exceeds the harmonic oscillator value by $60 \%$. The linear fitting of the momentum, $N_{b}=2$, which would be exact for the harmonic oscillator, recovers $86 \%$ of the difference, though the approximate wave function decays to the ground state slower than in the imaginary-time QM calculation using the splitoperator propagation. ${ }^{27}$ Increasing $N_{b}$ brings the trajectory calculation in agreement with the exact QM results within four significant figures for nearly all levels as summarized in Table I. The QM eigenvalues are obtained by the conventional Hamiltonian matrix diagonalization.

We studied the dependence of the approximate energies on the parameters of the initial wave function. The results presented in Table II show the average position, $\langle x\rangle / N$, variance, $\sigma=\left\langle x^{2}\right\rangle / N-\langle x\rangle^{2} / N^{2}$, and the normalized energy, $\bar{E}=\langle\hat{H}\rangle / N$ at final time $\tau=2$ a.u. for several values of the initial width parameter, $a=1 /\left(4 \sigma_{0}\right)$, and the Gaussian center, $x_{0}$. The initial energy, $\bar{E}_{0}$, indicates how different $\psi(x, 0)$ from the ground states is. The approximate quantum potential was derived from the linear, $N_{b}=2$, and cubic, $N_{b}=4$, fitting of the trajectory momenta. The initially displaced wavepackets take longer time to relax into the ground statetherefore, 1500 trajectories were propagated up to $\tau=2$ - but the calculations give the same value of energy within two significant digits for $N_{b}=2$ and four digits for $N_{b}=4$ bases. The final wavepacket localization, i.e., variance $\sigma_{\tau}$ is also well converged (three digits).

In general, the decay time and convergence to the ground state will be affected by the deviation of the initial wave function $\psi(x, 0)$ from the ground state wave function
TABLE II. Dependence of the ground state of the quartic oscillator given by Eq. (39) on the parameters of the initial wave function. Trajectories were propagated in imaginary time up to $\tau=2.0$ a.u. The quantum potential is obtained using $N_{b}=2$ and 4 basis functions to approximate the trajectory momenta. The listed quantities are defined in text.

\begin{tabular}{|c|c|c|c|c|c|c|c|c|}
\hline \multicolumn{3}{|c|}{ Initial $\psi$} & \multicolumn{3}{|c|}{$N_{b}=2$} & \multicolumn{3}{|c|}{$N_{b}=4$} \\
\hline$\sigma_{0}$ & $x_{0}$ & $\bar{E}_{0}$ & $\sigma_{\tau}$ & $\langle x\rangle_{\tau}$ & $\bar{E}_{\tau}$ & $\sigma_{\tau}$ & $\langle x\rangle_{\tau}$ & $\bar{E}_{\tau}$ \\
\hline 1.0 & 0.0 & 3.62 & 0.217 & 0.0 & 0.738 & 0.250 & 0.0 & 0.8034 \\
\hline 0.5 & 0.0 & 1.25 & 0.221 & 0.0 & 0.742 & 0.251 & 0.0 & 0.8034 \\
\hline 0.25 & 0.0 & 0.822 & 0.225 & 0.0 & 0.745 & 0.251 & 0.0 & 0.8034 \\
\hline 0.25 & 0.25 & 0.941 & 0.226 & -0.02 & 0.745 & 0.251 & 0.02 & 0.8034 \\
\hline 0.25 & 0.5 & 1.38 & 0.230 & -0.04 & 0.743 & 0.251 & 0.04 & 0.8034 \\
\hline
\end{tabular}

and by the values of the target energy levels. To obtain a converged highest energy state $(n=3$ in Table I) the projection procedure of Eq. (37) had to be performed very frequently taking time steps as small as $d \tau=10^{-4}$ a.u. This is not surprising since the wave function $\phi_{3}(x, \tau)$ $=\chi_{3}(x, \tau) \psi(x, \tau)$, extends into the region of higher anharmonicity than lower $n$ functions, and is the fastest to decay: if the lower energy components are not removed from the total wave function, which is a mixture of the eigenstates, this wave function will decay to a lower energy state. Nevertheless, it is certainly desirable to achieve better convergence of higher-energy states by improving the projection procedure.

Similar calculations have been performed for the Morse potential describing nonrotating $\mathrm{H}_{2}$ molecule multiplied by the reduced mass of $\mathrm{H}_{2}$ in atomic units

$$
V=D\left(e^{-z x}-1\right)^{2}-D_{0} .
$$

The parameter values are $D=160, z=1.0435 a_{0}^{-1}$, and $m=1$. The potential is shifted by $D_{0}=9$ to reduce decay of the wave functions. The initial width parameter, $a=9.2 a_{0}^{-2}$, and center position $x_{0}=0$, correspond to the harmonic approximation to the potential. The trajectories were propagated up to $\tau=0.2$ using $N_{b}=N_{c}=6$ basis, at which time all energy levels but $E_{3}$ reached plateau values. The convergence of the wave function energies to their respective energy levels is shown on Fig. 2 as relative deviations. We attribute the "drift" of $E_{3}$ to the rapid decay of $n=3$ state, i.e., even with the time step $d \tau=8 \times 10^{-6}$ the lower energy eigenstates are not fully projected out from $\phi_{3}(x, \tau)$. Reducing the time step by a factor of 2.5 increases the energy value to $E_{3}=58.33$, which changes its relative error from $-1.5 \%$ to $-0.06 \%$. Calculation of the lowest two energy states is much more robust: a time step $d \tau=4 \times 10^{-4}$ gives energy values converged within four significant figures.

The accuracy of the approximate trajectory results can also be assessed from the energy eigenvalues derived from Eq. (24). If $\phi_{n}$ decays to a single eigenstate with energy $E_{n}$, then

$$
\lim _{\tau \rightarrow \infty} \frac{d}{d \tau} \log N_{n}=-2 E_{n}
$$

The energy levels computed directly as in Eq. (36) and using Eq. (42) listed in Table III are in good agreement. 


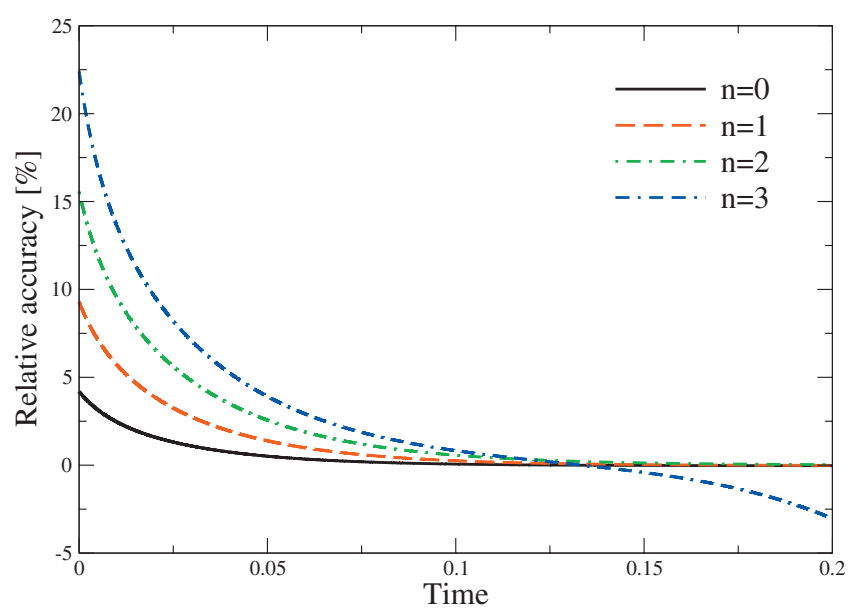

FIG. 2. Convergence of the energy levels for the Morse oscillator. Relative accuracy in percent is shown as a function of time. Imaginary-time QT dynamics and polynomial prefactors are implemented with the basis $N_{b}=N_{c}=6$.

The double well of Ref. 28 for several values of mass, $m=\{1,4,20\}$

$$
V=\left(x^{2}-1\right)^{2} / 4 \text {, }
$$

presents a more challenging application. As the mass is increased the ground state function changes from a single peak to a function with two maxima. The trajectory dynamics is more complicated, because unlike purely divergent trajectories of the single-well examples, for the heavy particle in the double well the central trajectories converge at the top of the barrier. The exact quantum trajectories are plotted on Figs. 3(a) and 3(b). Trajectory dynamics of the light particle, $m=1$, is similar to the single well potential: one can think that the effect of the quantum potential washes out the barrier region (or the central minimum of the inverted potential is 'filled in'). In case of the heavy particle, $m=20$, MDQP is small and behavior of the trajectories is more classical-like: trajectories originated in the central part, approximately with $|x(0)|<0.86$, converge to $x=0$ at large $\tau$; the outer trajectories launched from $|x(0)|>0.86$ roll off the inverted potential to negative infinity. In imaginary time the divergent trajectories (beyond Gaussian spreading) indicate building up of the wave function density and vice versa: $x=0$ region of Fig. 3 (b), where trajectories converge develops a wave function

TABLE III. The energy levels $(n=0,1,2,3)$ of the Morse oscillator obtained from the imaginary-time quantum trajectories propagated up to $\tau=0.18$. The size of the basis used to approximate $U$ and $\chi$ is $N_{b}=N_{c}=6$. The first two lines contain analytical harmonic approximation and the Morse oscillator results. $E_{\chi}$ are the energies of the approximate trajectory-based wave functions. $E_{N}$ indicates energies obtained from the decay of normalization from Eq. (42). The energies are given in scaled units as described in text. The bottom line contains wave function norms at $\tau=0.18$.

\begin{tabular}{lcccc}
\hline \hline & $E_{0}$ & $E_{1}$ & $E_{2}$ & $E_{3}$ \\
\hline $\mathrm{HO}$ & 9.33 & 28.00 & 46.67 & 65.33 \\
$\mathrm{QM}$ & 9.20 & 26.78 & 43.26 & 58.66 \\
$E_{\chi}$ & 9.20 & 26.78 & 43.28 & 57.76 \\
$E_{N}$ & 9.20 & 26.78 & 43.33 & 57.87 \\
$N$ & 0.91 & $1.5 \times 10^{-3}$ & $3.2 \times 10^{-6}$ & $9.1 \times 10^{-8}$ \\
\hline \hline
\end{tabular}
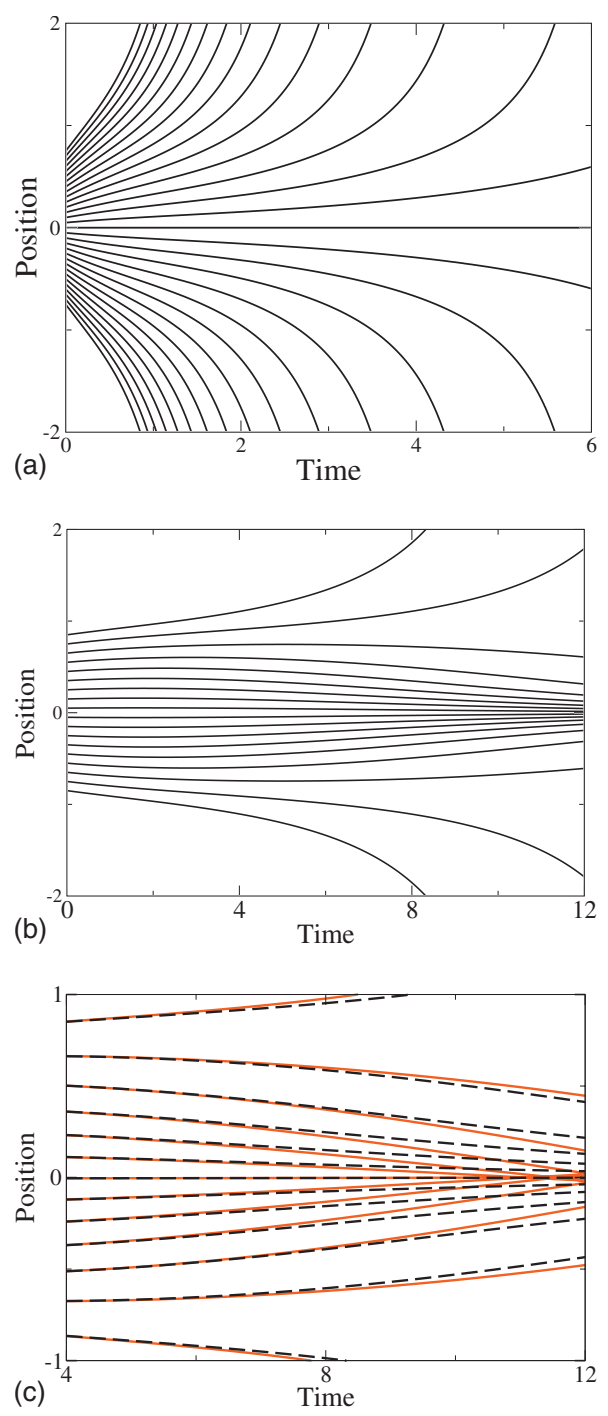

FIG. 3. Exact imaginary time quantum trajectories for the double well potential computed for the light $(m=1)$ and heavy $(m=20)$ particles shown on panels [(a) and (b)], respectively. Panel (c) shows exact QM (dash) and approximate (solid line) trajectories for the heavy particle. $N_{b}=4$ was used in the approximate dynamics.

minimum; maxima develop around $x= \pm 0.86$, where trajectories diverge. Another notable feature is that exact quantum trajectories converging at $x=0$ do not cross - the exponential representation of a positive wave function Eq. (14) remains single-valued and unique at all times.

The noncrossing trajectory behavior is difficult to reproduce numerically in imaginary (or real) time. As a result, the Taylor basis approximation to the trajectory momenta for the heavy particle in the double well is not as accurate and the energy levels are not as converged as in other examples. The exact and approximate $\left(N_{b}=4\right)$ trajectories are shown on Fig. 3(c). The approximate trajectories cross at $\tau>10$ a.u. Nevertheless, our dynamics approach and computation of average quantities, both based on trajectory weights, are insensitive to these crossings and numerically stable even if inaccurate. Moreover, imaginary-time dynamics of a trajectory ensemble is insensitive to inaccuracies of large action functions, as their contributions exponentially depend on $S(x, \tau)$. Using larger bases, in principle, should 
TABLE IV. The energy levels for the double well for $n=0,1,2,3$ obtained with the imaginary-time approximate QTs and from the Hamiltonian diagonalization $(\mathrm{QM})$. The initial width parameter is $a=0.75$ for all values of $m$. The normalized energies of functions $\phi_{n}$ are computed at time $\tau$ listed in the second column.

\begin{tabular}{lcccccc}
\hline \hline $\begin{array}{l}m \\
(\text { a.u. }\end{array}$ & $\begin{array}{c}\tau \\
(\text { a.u. })\end{array}$ & Method & $E_{0}$ & $E_{1}$ & $E_{2}$ & $E_{3}$ \\
\hline 1 & 2.0 & QT & 0.397 & 1.122 & 2.395 & 3.842 \\
& & QM & 0.397 & 1.122 & 2.378 & 3.841 \\
4 & 3.0 & QT & 0.218 & 0.415 & 0.899 & 1.421 \\
& & QM & 0.217 & 0.415 & 0.886 & 1.416 \\
20 & 8.0 & QT & 0.133 & 0.152 & 0.345 & 0.473 \\
& 10.0 & QT & 0.131 & 0.152 & 0.348 & 0.479 \\
& & QM & 0.133 & 0.152 & 0.332 & 0.465 \\
\hline \hline
\end{tabular}

give converged eigenvalues, but can be impractical and unstable in the approximate implementation. Since our goal is the development of a cheap method scalable to multidimensional systems, we did not pursue full QM implementation with large basis sets.

Table IV shows four lowest energy levels computed with $N_{b}=N_{c}=6$. The initial width parameter is $a=0.75 a_{0}^{-2}$ for all calculations. We attribute the discrepancies to the errors in the quantum force: Eq. (42), indicating that the wave function decayed into an eigenstate, is not fulfilled. [At the same time, Eq. (24) is fulfilled with high accuracy.] The agreement of the wave function energies with the exact QM energy levels is still fairly good, including $E_{1}-E_{0}$ energy splitting of 0.02 a.u. for the largest mass. The ground state wave functions for $m=1$ and $m=20$ are shown on Fig. 4. Note that the double-peak density for $m=20$ has evolved from the initial Gaussian function in the course of the imaginary time evolution. With real-time quantum trajectories we had to resort to polynomial $\chi$-prefactors to reproduce such density. ${ }^{16}$

\section{CONCLUSIONS}

Advantages of the trajectory-based approaches to QM problems are the intuitive visualization of the trajectory dynamics and the linear scaling of the propagation cost with dimensionality (not considering the number of trajectories) at the expense of making approximations. The QT dynamics provides a framework for an approximate trajectory methodology describing the dominant quantum effects. Since for large molecular systems one is often interested in correlation functions at a given temperature, it is desirable to extend the QT approach to evolution of wave functions in imaginary time, which is equivalent to evolution with the Boltzmann operator, which can be followed by the real-time dynamics.

We have described the imaginary-time adaptation of the quantum-trajectory dynamics whose distinct features are: (i) the trajectory momentum is defined by the action function, $p=\nabla S$, as in real-time Bohmian dynamics, (ii) a positive wave function is represented as a simple exponential function, $\psi(x, \tau)=\exp (-S(x, \tau))$, and (iii) the momentumdependent quantum potential (MDQP), $U=\hbar \nabla p /(2 m)$, replaces the amplitude-dependent quantum potential of the real-time SE. The first feature makes the new formulation

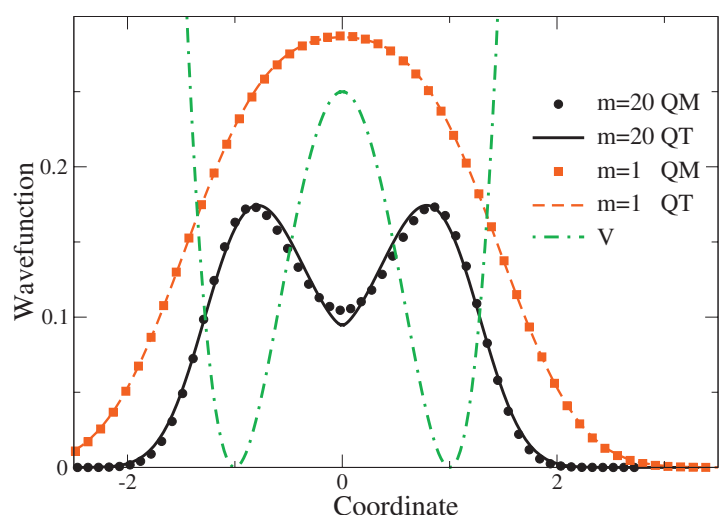

FIG. 4. The ground state of the double well for $m=20$ and $m=1$ after propagation up to $\tau=10$ a.u. and $\tau=2$ a.u., respectively, computed with the bases of size $N_{b}=N_{c}=6$. Symbols indicate QM wave functions obtained with the split-propagator evolution. The QT results are shown with lines. The classical potential is represented with the dash.

different from ZEVCA, ${ }^{21}$ where "trajectories" do not move by definition. The second feature makes the MDQP formulation different from the formalism of Makri and Liu, ${ }^{20}$ where the wave function is repeatedly repartitioned between the amplitude and the phase-containing factors to achieve stable, essentially stationary, trajectories. The approximate implementation of MDQP also differs from the semiclassical implementation of ZEVCA and from the imaginary-time Bohmian trajectory stability (BTS) approach. ${ }^{20}$ We have described a methodology based on the evolution of the trajectory ensemble with the quantum potential and force derived from the moments of the trajectory distribution. Only the first derivatives of the classical potential are needed, because the region of $V$ relevant to the problem, such as the low energy region in eigenstate calculations, is explored by multiple trajectories. In BTS and ZEVCA one can use a single zero-velocity trajectory, but has to evolve stability matrix elements and their derivatives requiring high-order derivatives of $V$. A single trajectory calculation gives the lowest energy of specified symmetry (reconstruction of a wave function requires multiple trajectories). Effectively in this case information about the low-energy region of $V$ is obtained from the Taylor expansion of $V$ near its minimum instead of exploring this region with multiple trajectories. The single trajectory framework might be useful in computation of the vibrational zero-point energies of highly localized energy eigenstates. The trajectory-ensemble framework should be better suited do describe more diffuse wave functions, such as the heavy particle eigenstate in a double well, or for scattering dynamics, such as in computation of the thermal reaction rate constants. Combination of imaginarytime ZEVCA or BTS and MDQP trajectories, similar to realtime Lagrangian-Eulerian grids of Trahan and Wyatt, ${ }^{29}$ might be also useful in vibrational calculations.

Our approximate implementation of MDQP was based on the global Least-squares Fit of the trajectory momenta. A Gaussian wave function, whose initial width parameter in our formulation defines initial actions and momenta of the trajectories, the momentum is linear, $U$ is a time-dependent constant producing zero quantum force. For general func- 
tions, a contribution of the quantum potential to average quantities is compensated by the change in the trajectory volume element, which is never computed explicitlytrajectory contributions depend on the classical action function computed along the quantum trajectory. For a cheap global implementation - a single approximation works for the entire trajectory ensemble-the quantum potential is found by fitting the trajectory momenta in terms of a small (2-6 functions) polynomial basis. A nodeless wave function decays to the ground energy state. The same basis, used to define polynomial prefactors or $\chi$-functions, was utilized to evolve wave functions with nodes, which decay to the excited energy states provided that the lower energy contributions are projected out. As a proof-of-concept four lowest energy eigenstates were computed for anharmonic singlewell potential and for the double well. The Taylor basis of six functions gave accurate (four significant figures) results for the single wells. For the more challenging double-well problem, the accuracy and convergence were not as good, but the energy splitting due to tunneling, which is about $1 / 8$ of the zero-point energy, was accurately reproduced. Moreover, the two maxima of the ground state wave function emerged from the evolution of the initial Gaussian function, something that is very challenging to reproduce with quantum trajectories in real time.

We expect that in many dimensions the approximate MDQP can give cheap estimates of quantum effects on dynamics of nuclei, reasonably described by classical mechanics. For such systems low order approximations $N_{b}=2$, perhaps $N_{b}=4$ for strongly anharmonic modes, will give accurate QM corrections. In case of $N_{b}=2$ the size of the multidimensional basis will scale linearly with dimensionality and inversion of a matrix of the size $\left(N_{\mathrm{dim}}+1\right) \times\left(N_{\mathrm{dim}}\right.$ $+1)$ will be the most expensive step of MDQP calculation, performed at each time-step but just once for all trajectories. For realistic chemical systems the calculation is likely to be dominated by the computation of classical forces. Issues of accuracy, cost, and scaling will be explored in our future work focused on high-dimensional applications and combi- nation of imaginary-time QT evolution with real-time dynamics for calculations of the reaction rate constants.

\section{ACKNOWLEDGMENTS}

This work was supported by the University of South Carolina.

${ }^{1}$ S. Janecek and E. Krotscheck, Comput. Phys. Commun. 178, 835 (2008).

${ }^{2}$ D. Blume, M. Lewerenz, P. Niyaz, and K. B. Whaley, Phys. Rev. E 55, 3664 (1997).

${ }^{3}$ D. M. Ceperley and L. Mitas, in New Methods in Computational Quantum Mechanics, Advances in Chemical Physics Vol. 93, edited by I. Prigogine and S. A. Rice (Wiley, New York, 1996).

${ }^{4}$ W. A. Lester, Jr., L. Mitas, and B. Hammond, Chem. Phys. Lett. 478, 1 (2009).

${ }^{5}$ A. Viel, M. D. Coutinho-Neto, and U. Manthe, J. Chem. Phys. 126, 024308 (2007).

${ }^{6}$ C. E. Hinkle and A. B. McCoy, J. Phys. Chem. A 112, 2058 (2008)

${ }^{7}$ N. Makri and W. H. Miller, J. Chem. Phys. 116, 9207 (2002).

${ }^{8}$ W. H. Miller, S. D. Schwartz, and J. W. Tromp, J. Chem. Phys. 79, 4889 (1983).

${ }^{9}$ S. Garashchuk and V. A. Rassolov, Chem. Phys. Lett. 364, 562 (2002).

${ }^{10}$ A. Donoso, Y. J. Yeng, and C. C. Martens, J. Chem. Phys. 119, 5010 (2003).

${ }^{11}$ J. B. Maddox and E. R. Bittner, J. Chem. Phys. 119, 6465 (2003).

${ }^{12}$ J. Liu and N. Makri, J. Phys. Chem. A 108, 5408 (2004).

${ }^{13}$ C. J. Trahan, K. Hughes, and R. E. Wyatt, J. Chem. Phys. 118, 9911 (2003)

${ }^{14}$ S. Garashchuk and V. A. Rassolov, J. Chem. Phys. 120, 1181 (2004).

${ }^{15}$ S. Garashchuk and V. A. Rassolov, J. Chem. Phys. 121, 8711 (2004).

${ }^{16}$ S. Garashchuk, J. Phys. Chem. A 113, 4451 (2009).

${ }^{17}$ E. Madelung, Z. Phys. 40, 322 (1926).

${ }^{18}$ D. Bohm, Phys. Rev. 85, $166(1952)$.

${ }^{19}$ W. H. Miller, J. Chem. Phys. 55, 3146 (1971).

${ }^{20}$ J. Liu and N. Makri, Mol. Phys. 103, 1083 (2005).

${ }^{21}$ Y. Goldfarb, I. Degani, and D. J. Tannor, Chem. Phys. 338, 106 (2007).

${ }^{22}$ Y. Goldfarb, I. Degani, and D. J. Tannor, J. Chem. Phys. 125, 231103 (2006).

${ }^{23}$ W. H. Press, B. P. Flannery, S. A. Teukolsky, and W. T. Vetterling, Numerical Recipes: The Art of Scientific Computing, 2nd ed. (Cambridge University Press, Cambridge, 1992).

${ }^{24}$ E. R. Bittner, J. Chem. Phys. 119, 1358 (2003).

${ }^{25}$ V. A. Rassolov and S. Garashchuk, J. Chem. Phys. 120, 6815 (2004).

${ }^{26}$ S. Garashchuk and V. A. Rassolov, J. Chem. Phys. 129, 024109 (2008).

${ }^{27}$ M. D. Feit, J. A. Fleck, Jr., and A. Steiger, J. Comput. Phys. 47, 412 (1982).

${ }^{28}$ D. Blume, M. Lewerenz, and K. B. Whaley, J. Chem. Phys. 107, 9067 (1997).

${ }^{29}$ C. J. Trahan and R. E. Wyatt, J. Chem. Phys. 118, 4784 (2003). 